\title{
ON THE HOLOMORPHIC CURVATURE OF SOME INTRINSIC METRICS
}

\author{
B. WONG
}

\begin{abstract}
If $G$ is a hyperbolic manifold in the sense of Kobayashi and the differential Kobayashi metric $K_{G}$ is of class $C^{2}$, then the holomorphic curvature of $K_{G}$ is greater than or equal to -4 . If $G$ is Carathéodory-hyperbolic and the differential Caratheodory metric $C_{G}$ is of class $C^{2}$, then the holomorphic curvature of $C_{G}$ is less than or equal to -4 . With this result we obtain an intrinsic characterization of the unit ball.
\end{abstract}

\section{Introduction.}

(A) Definition. Let $G$ be a complex manifold and $T(G)$ the tangent bundle; we define the differential Carathéodory metric as follows: $C_{G}$ : $T(G) \rightarrow R^{+} \cup\{0\}$,

$$
C_{G}(z, v)=\sup _{f}\{|d f(v)|: f \in D(G), f(z)=0\},
$$

where $D(G)$ denotes the set of holomorphic mappings from $G$ to the unit disc $D$, and the norm of $d f(v)$ is taken with respect to the Poincare metric in $D$.

$G$ is defined to be Carathéodory-hyperbolic if $C_{G}$ is nontrivial everywhere.

The differential Kobayashi metric is defined as follows: $K_{G}: T(G) \rightarrow R^{+}$ $\cup\{0\}$,

$$
\begin{aligned}
K_{G}(z, v)=\inf _{f}\left\{|t|: t \in T_{0}(D),\right. & \text { and } f \in G(D) \\
& \text { such that } f(0)=z, d f(t)=v\},
\end{aligned}
$$

where $G(D)$ denotes the set of holomorphic mappings from $D$ to $G$, and the norm is taken with respect to the Poincaré metric in $D . G$ is defined to be hyperbolic if $K_{G}$ is nontrivial everywhere.

For a standard reference of this area one can consult Kobayashi [4] (or Wu [12]).

(B) Hermitian Finsler metric and holomorphic curvature.

Definition Suppose that $G$ is a complex manifold. Then $F: T(G) \rightarrow R^{+} \cup$ $\{0\}$ is a hermitian Finsler metric if

(1) $F(z, s v)=|s| \cdot F(z, v)$, where $s \in C$,

(2) $F(z, v)=0$ iff $v=0$, for any $z \in G$.

Received by the editors April 16, 1976 and, in revised form, May 13, 1976, August 13, 1976, and September 14, 1976.

AMS (MOS) subject classifications (1970). Primary 32H15, 32H20, 32H25; Secondary 32A15, 32A17, 32C10, 32E10.

Key words and phrases. Kobayashi metric, Carathéodory metric, holomorphic curvature. 
Suppose that $F$ is a $C^{2}$ hermitian Finsler metric on a complex one dimensional manifold. It is obvious that in this case $F$ is just a $C^{2}$ hermitian metric in the usual sense of differential geometry. Then the holomorphic curvature of $F$ is given by the following formula:

$$
K(F)=-\frac{1}{F} \frac{\partial^{2} \log F}{\partial z \partial \bar{z}} .
$$

Let $G$ be a complex manifold as before and $M_{P}(v)$ any complex one dimensional submanifold through the point $P$ and whose tangent space at $P$ is spanned by $\{v, J v\}$. In the following, $G(v)_{P}$ is the set of all $M_{P}(v)$.

Definition. The holomorphic curvature $k_{F}(P, v)$ of a $C^{2}$ hermitian Finsler metric $F$ at $(P, v) \in T(G)$ is defined to be the following number:

$$
\begin{aligned}
& k_{F}(P, v) \sup _{G(v)_{P}}\{\text { the holomorphic curvature of the } \\
&\text { restriction of } \left.F \text { to } M_{P}(v)\right\} .
\end{aligned}
$$

REMARK. If $F$ is a standard hermitian metric, then our definition coincides with the usual one. The proof of this assertion follows immediately from $\mathrm{Wu}$ [13, Lemma 1, p. 1105 and Lemma 4, p. 1106].

2. Statement of results.

THeOREM 1. (A) If $G$ is Carathéodory-hyperbolic and $C_{G}$ is of class $C^{2}$, then the holomorphic curvature of $C_{G}$ is less than or equal to -4 .

(B) If $G$ is hyperbolic in the sense of Kobayashi, and $K_{G}$ is of class $C^{2}$, then the holomorphic curvature of $K_{G}$ is greater than or equal to -4 .

THEOREM 2. Let $G$ be a simply connected bounded domain in $C^{n}$. Assume that

(a) $G$ is complete hyperbolic,

(b) $K_{G}=C_{G}$, and they are smooth hermitian metrics in the usual sense of differential geometry.

Then $G$ is biholomorphic to the unit ball.

3. Proof. (A) Carathéodory; (B) Kobayashi; (C) Theorem 2.

(A) Let us fix a tangent $(P, v)$ at $P$. It is clear from Definition $1(\mathrm{~B})$ that it suffices to prove the holomorphic curvature of the restriction of $C_{G}$ to any $M_{P}(v)$ of $G(v)_{P}$ is less than -4 .

We need the following lemma to prove both part (A) and part (B).

LEMMA. Let $M, N$ be complex manifolds, and $N$ complete hyperbolic in the sense of Kobayashi, suppose that we fix two points $x_{1}$ and $x_{2}$ in $M$ and $N$ respectively. Then $S=\left\{f \in \operatorname{Hol}(M, N): f\left(x_{1}\right)=x_{2}\right\}$ is compact in $\operatorname{Hol}(M, N)$ with respect to the compact open topology.

(See [4] or [12] for the proof.)

Making use of the compactness of $S$, one can obtain a mapping $f$ belonging to $\operatorname{Hol}(G, D)$ satisfying the following conditions:

(1) $f(P)=0$ and

(2) $C_{G}(P, v)=\left|d f_{P}(v)\right|$. 
We observe that $d f_{P} \neq 0$. For any $M_{P}(v)$ one can choose a neighborhood $U$ of the origin 0 in $D$ such that $f: M_{P}(v) \rightarrow U$ is a biholomorphism (for sufficiently small choice of $M_{P}(v)$ ).

With respect to the local coordinates $\{z, \bar{z}\}$ of $M_{P}(v), f^{*}\left(B_{D}\right)$ (the pullback of $B_{D}$ by $f$ restricted to $\left.M_{P}(v)\right)$ and the restriction of $C_{G}$ to $M_{P}(v)$ can be written as follows:

The restriction of $f^{*}\left(B_{D}\right)$ to $M_{P}(v)=h \cdot d z \otimes d \bar{z}$;

The restriction of $C_{G}$ to $M_{P}(v)=g \cdot d z \otimes d \bar{z}$, where $h$ and $g$ are smooth functions on $M_{P}(v)$.

It is important to point out here that the Carathéodory metrics enjoy the distance decreasing property under holomorphic mappings. Therefore we have the following inequality: $f^{*}\left(B_{D}\right) \leqslant C_{G}$ (i.e. $h \leqslant g$ ).

We let $u=h / g$. From (2) we have $h(P)=g(P)\left(f\right.$ realizes $C_{G}$ at the point $P)$. Together with the above inequality $\left(h \leqslant g\right.$ on $\left.M_{P}(v)\right)$ and the definition of $u$, one can obtain the following two conditions of $u$ :

(a) $u(P)=1$ (i.e. $\log u(P)=0$ ),

(b) $u \leqslant 1$ on $M_{P}(v)$ (i.e. $\log u \leqslant 0$ ).

This means $\log u$ attains a maximum at $P$. Therefore we have

$$
\left(\partial^{2} / \partial z \partial \bar{z}\right)(\log u)(P) \leqslant 0 .
$$

From the fact that $\log u=\log h-\log g$, we have the following inequality:

$$
\left(\partial^{2} / \partial z \partial \bar{z}\right)(\log h)(P) \leqslant\left(\partial^{2} / \partial z \partial \bar{z}\right)(\log g)(P) .
$$

However, since $h(P)=g(P)$, we easily get

$$
-\frac{1}{h} \frac{\partial^{2}}{\partial z \partial \bar{z}}(\log h)(P) \geqslant-\frac{1}{g} \frac{\partial^{2}}{\partial z \partial \bar{z}}(\log g)(P),
$$

The left-hand side is just the holomorphic curvature of the Poincare metric on $D$, which is equal to -4 . The right-hand side is the holomorphic curvature of the restriction of $C_{G}$ to $M_{P}(v)$. This completes the proof.

(B) Let us fix $(P, v)$ in $T(G)$. It is clear from our definition of holomorphic curvature that Theorem 1(B) would follow if one could prove there exists one $M_{P}(v)$ whose holomorphic curvature of the restriction of $K_{G}$ to $M_{P}(v)$ at the point $P$ is greater than -4 .

From the definition of the differential Kobayashi metric there exists a sequence of holomorphic functions $\left\{f_{i}\right\} \subset \operatorname{Hol}(D, G)$, such that $f_{i}(0)=$ $P, K(P, v)=\lim _{i \rightarrow \infty}\left|t_{i}\right|$, where $d f_{i}\left(t_{i}\right)=v, t_{i}$ is a tangent at the origin of $D$, and $\left|t_{i}\right|$ is taken with respect to the Poincaré metric in the unit disc $D$.

$G$ is assumed to be hyperbolic in the sense of Kobayashi, so that it is a tight manifold in the sense of $\mathrm{Wu}$ (see [12]). Therefore there exist neighborhoods $U_{1}, U_{2}$ of 0 and $P$, respectively, such that $f_{i}\left(U_{1}\right) \subset U_{2}$ for all $i$. Furthermore, $U_{2}$ can be chosen to be complete hyperbolic (for example, biholomorphic to the unit ball).

Applying the lemma in part (A) again, we obtain a holomorphic mapping $f$ : $U_{1} \rightarrow U_{2}$ satisfying the following conditions. (We consider $S$ to be the set of 
holomorphic mappings $\left\{f_{i}\right\}$ from $U_{1}$ to $U_{2}$. As $U_{2}$ is chosen to be complete hyperbolic, we can apply the compactness of $S$ to obtain $f$.)

(a) $f(0)=P$.

(b) $d f_{0}(t)=v$, for a tangent vector $t$ of $D$ at the origin 0 , and $|t|=$ $K_{G}(P, v)$.

(c) $B_{D}(z, w) \geqslant K_{G}\left(f(z), d f_{0}(w)\right)$, for all $(z, w) \in T(D), z_{1} \in U_{1}$.

((a) and (b) are trivial. For (c) one has to observe the fact that $B_{D}(z, w) \geqslant$ $K_{G}\left(f_{i}(z), d f_{i}(w)\right)$ for all $i$. $\left\{f_{i}\right\}$ converges to $f$ with respect to the compact-open topology in $\operatorname{Hol}\left(U_{1}, U_{2}\right)$, so it is easy to see the inequality holds in the limit.)

We can choose a sufficiently small neighborhood $U \subset U_{1}$ of the origin 0 , such that $f(U)=M_{P}(v)$ is a complex manifold whose tangent space is spanned by $\{v, \bar{v}\}$. With respect to the coordinates $\{z, \bar{z}\}$ of $U$, one can write:

$g \cdot d z \otimes d \bar{z}=$ the pullback of the restriction of $K_{G}$ to $M_{P}(v)$ by $f$,

$h \cdot d z \otimes d \bar{z}=$ the Poincare metric of the unit disc $D$.

We let $u=h / g$. From (a), (b) and (c) we can obtain the following:

(1) $u(0)=1$. (I.e. $\log u(0)=0$. This follows from the fact that $f$ realizes $K_{G}(P, v),(\mathrm{b})$.)

(2) $u \geqslant 1$ on $U$. (I.e. $\log u \geqslant 0$ on $U$. This follows from the fact that Kobayashi metrics are distance-decreasing under holomorphic mappings. (c) simply means $h \geqslant g$ on $U$; therefore $u \geqslant 1$.)

Clearly $\log u$ attains a minimum at 0 in $U$. Hence we have the following inequalities:

$$
\begin{aligned}
\left(\partial^{2} / \partial z \partial \bar{z}\right)(\log h)(0) & \geqslant 0, \\
-\frac{1}{h} \frac{\partial^{2}}{\partial z \partial \bar{z}}(\log h)(0) & \leqslant-\frac{1}{g} \frac{\partial^{2}}{\partial z \partial \bar{z}}(\log g)(0) .
\end{aligned}
$$

(In order to obtain the second inequality one has to use the fact $h(0)=$ $g(0)$.)

One observes that the left-hand side of the above second inequality is the holomorphic curvature of the Poincaré metric, which is identically equal to -4. The right-hand side is equal to the holomorphic curvature of the restriction of $K_{G}$ to $M_{P}(v)$ at the point $P$.

Our proof is therefore completed.

(C) The following theorem is well known.

THEOREM. Any simply connected complete Kähler manifold with constant negative holomorphic curvature is biholomorphic to the unit ball.

The following lemma is also well known in differential goemetry.

LEMMA. Any hermitian manifold with nonzero constant holomorphic curvature is Kähler.

Suppose that $K_{G}=C_{G}$, and they are hermitian metrics. From Theorem 1 and the above lemma we can conclude that $K_{G}$ and $C_{G}$ are Kähler metrics in $G$. By the assumption that $G$ is complete hyperbolic our theorem follows 
immediately. (Note: By the theorem of Royden [6], Cauchy completeness of $K_{G}$ is equivalent to the finite compactness. If $K_{G}$ is Kähler, then complete hyperbolic implies the completeness in the usual sense of Riemannian geometry.)

4. Some final remarks. We have to point out that the $C^{2}$ condition on $K_{G}$ and $C_{G}$ is a very unnatural assumption. In most cases they are only continuous or semicontinuous. This artificial assumption is due to the technical difficulties of defining the holomorphic curvature. We believe that if one could define the holomorphic curvature of a semicontinuous hermitian metric in a proper way, our result would equally hold. (A formula of holomorphic curvature was suggested in our former manuscript, On the holomorphic curvature of the Kobayashi metrics and Carathéodory metrics.)

The following question is fundamental in hyperbolic complex analysis. If $G$ is complete hyperbolic, does $K_{G}$ satisfy the maximum modulus principle in $T(G)$ ?

I have to thank Griffiths, Kobayashi, Paul Yang, and S. T. Yau for many interesting conversations.

Burbea informed us that he had a similar result on the Hessian of the Carathéodory metric.

\section{REFERENCES}

1. B. A. Fuks, Special chapters in the theory of analytic functions of several complex variables, Fizmatgiz, Moscow, 1963; English transl, Transl. Math. Monographs, vol. 14, Amer. Math. Soc., Providence, R. I., 1965. MR 30\#4979; 32 \#915.

2. I. Graham, Boundary behavior of the Carathéodory and Kobayashi metrics on strongly pseudoconvex domains in $C^{n}$ with smooth boundary, Trans. Amer. Math. Soc. 207 (1975), 219-240. MR 51 \# 8468.

3. S. Kobayashi, Geometry of bounded domains, Trans. Amer. Math. Soc. 92 (1959), 267-290. MR 22 \#3017.

4. 43 \#3503.

5. Some remarks on the questions concerning intrinsic distance, Tôhoku Math. J. (2) 25 (1973), 481-486. MR 48 \#8856.

6. H. L. Royden, Remarks on the Kobayashi metric, Several Complex Variables. II, Lecture Notes in Math., vol. 185, Springer, Berlin, 1971, pp. 125-137. MR 46\#3826.

7. H. Rund, The differential geometry of Finsler spaces, Grundlehren math. Wiss., Band 101, Springer-Verlag, Berlin, 1959. MR 21 \#4462.

8. Hans-Jörg Reiffen, Die differentialgeometrischen Eigenschaften der invarianten Distanzfunktion von Carathéodory, Schr. Math. Inst. Univ. Münster No. 26 (1963). MR 28 \# 1320.

9. Die Carathéodorysche Distanz und ihre zugehörige Differentialmetrik, Math. Ann. 161 (1963), 315-324. MR 33 \#4325.

10. B. Wong, Scalar curvature of some intrinsic measures (preprint).

11. The boundary behavior of some intrinsic measures on the strongly pseudo-convex bounded domains with smooth boundary (to appear).

12. H. Wu, Normal families of holomorphic mappings, Acta Math. 119 (1967), 193-233. MR 31 \#468.

13. _ A remark on holomorphic sectional curvature, Indiana Univ. Math. J. 22 (1972/73), 1103-1108. MR 47 \#4191. 